\section{COMPARISON OF THE EFFECT OF MONOPOLAR AND BIPOLAR CAUTERIZATION ON SKELETONIZED, DISSECTED INTERNAL THORACIC ARTERIES}

The internal thoracic artery is preferable to the saphenous vein for use as a conduit for coronary artery bypass. More extensive use is possible if this artery is mobilized in a skeletonized form to provide greater length. Internal thoracic arteries are usually mobilized with cauterization. This study compared the effectiveness and effects on neighboring areas of division of the branches of the canine internal thoracic artery with bipolar cauterization and monopolar cauterization. Branch closure was significantly more secure in the bipolar cauterization group, with bleeding in 25 (9\%) of 279 branches of 15 internal thoracic arteries treated with monopolar cauterization, in contrast to bleeding in $4(1.3 \%)$ of 306 branches in the 15 internal thoracic arteries treated with bipolar cauterization, which were paired with the group treated with monopolar cauterization. The group of internal thoracic arteries treated with monopolar cauterization had a significantly higher prevalence of leakage when luminal pressure was increased from 120 to $160 \mathrm{~mm} \mathrm{Hg}$. Scanning electron microscopy demonstrated partial loss of endothelial cells on the flow surface of internal thoracic arteries treated with bipolar cauterization, compared with almost complete Ioss of endothelial cells around the orificial areas after monopolar cauterization. Secondary bipolar cauterization treatment caused only slightly more damage than primary treatment, but secondary monopolar cauterization was much more severe and extensive than primary treatment. These data suggest that bipolar cauterization is preferable to monopolar cauterization for skeletonized dissection of the internal thoracic artery. (J Thorac Cardiovasc Surg 1995;110:504-10)

Hiroki Yoshida, MD, Moses Hong-De Wu, MD, Yasuhiro Kouchi, MD, Yoko Onuki, MD, Qun Shi, MD, and Lester R. Sauvage, MD, Seattle, Wash.
$S^{t a t i n s}$ tudies have shown that the internal thoracic artery (ITA) used as a coronary artery bypass graft has a long-term result that is significantly superior to results obtained with the saphenous vein. ${ }^{1,2}$ We have developed techniques to completely revascularize the entire coronary artery system with only the ITAs. ${ }^{3,4}$ This requires maximum lengths of the ITA, with skeletonized mobilization. Most surgeons dissect the ITA as a pedicle graft, or as a free graft with surrounding tissue, with the use of monopolar cauterization (MPC) for hemostasis, reinforced as needed by hemoclips. ${ }^{5,6}$ Although this method provides a graft sufficiently long for a left

From The Hope Heart Institute, the Providence Medical Center, and the Department of Surgery, University of Washington School of Medicine, Seattle, Wash.

Received for publication Sept. 2, 1994.

Accepted for publication Dec. 28, 1994.

Address for reprints: Lester R. Sauvage, MD, 528 18th Ave., Seattle, WA 98122.

Copyright (C) 1995 by Mosby-Year Book, Inc.

0022-5223/95\$3.00+0 $\mathbf{1 2 / 1 / 6 3 1 7 9}$
ITA graft to the left anterior descending artery, it may not yield one that can reach the distal right coronary artery or its proximal posterior descending or left ventricular extension branches. Some reports have mentioned surgical methods of ITA graft mobilization, including skeletonized dissection ${ }^{5-8}$; however, there has been little description of the type of cauterization used and the effect of its application. In 1987 Keeley $^{9}$ noted that bipolar cauterization (BPC) allows precise control of current and avoids random spraying of heat. For the past several years we have found that mobilization of the ITAs in a skeletonized manner is facilitated by use of BPC. This study was undertaken to compare the results of mobilization of the ITAs with MPC versus BPC, with special attention paid to the quality and security of branch closure and the extent and degree of tissue damage.

\section{Material and methods}

Care of the dogs used in this study complied with the "Principles of Laboratory Animal Care" and the "Guide 


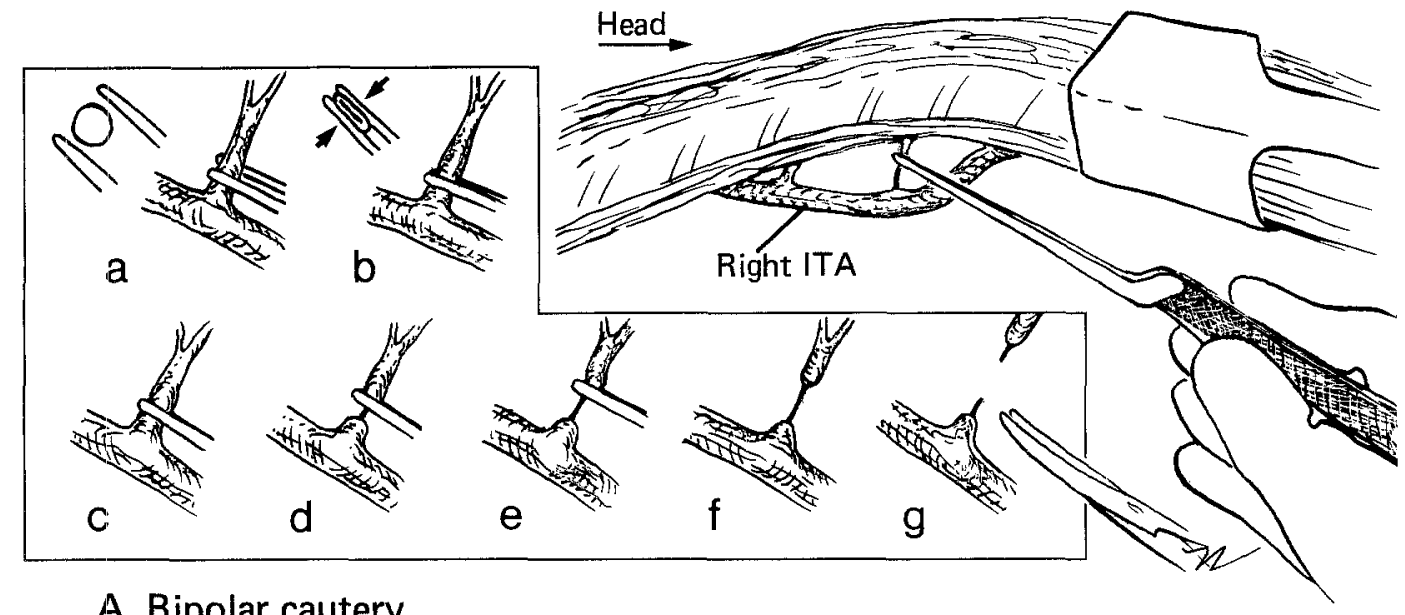

\section{A. Bipolar cautery}

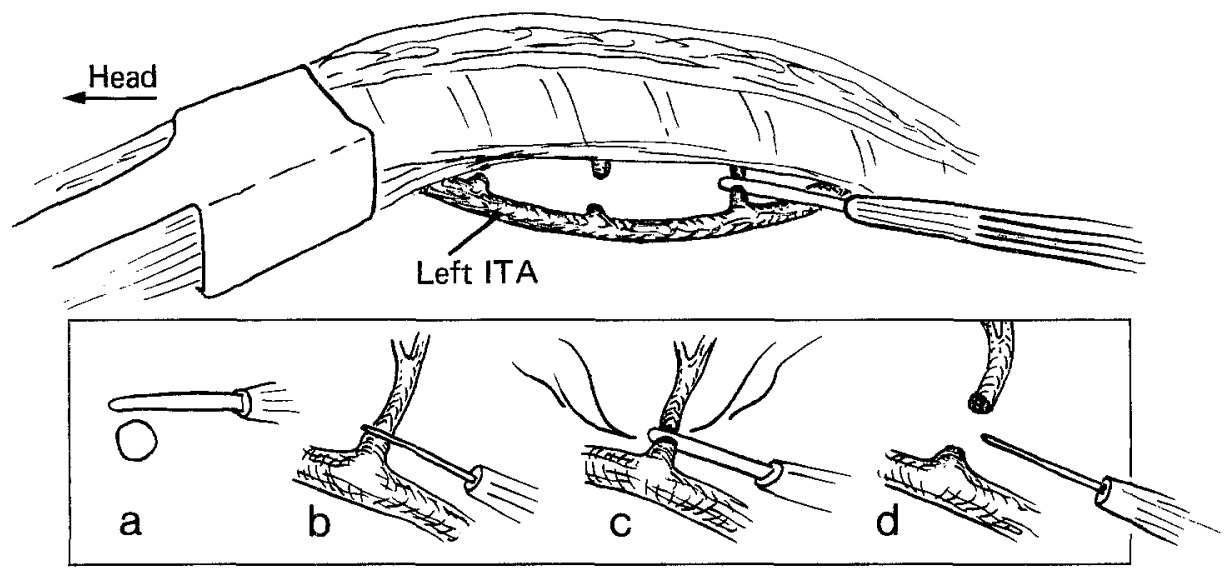

\section{B. Monopolar cautery}

Fig. 1. Surgical technique for dissecting out ITA with BPC (A) and MPC (B) ( $a$ to $g$ and $a$ to $d$ indicate sequence).

for the Care and Use of Laboratory Animals" (National Institutes of Health Publication No. 80-23, revised 1985). Seventeen randomly selected dogs weighing from 21.0 to $37.0 \mathrm{~kg}$ (average $23.6 \pm 3.7 \mathrm{~kg}$ ) were studied in a paired comparison form, in which one ITA was dissected by MPC and the contralateral ITA was dissected by BPC; the sides were alternated by cases. The surgical dissection was done with the use of proper anesthesia, which was induced with 5 to $10 \mathrm{ml} 4 \%$ thiamylal intravenously and maintained with a combination of $0.5 \%$ to $1 \%$ halothane and a mixture of nitrous oxide and oxygen in a $2: 1$ ratio via an endotracheal tube connected to a closed-circuit respirator.

The chest was opened through a median sternotomy to allow access to both ITAs for skeletonized dissection. The cautery unit used in this study was a Bard System 5000 (Davol Electro Medical System, Englewood, Colo.), which can perform both MPC and BPC in either a cutting or a coagulant mode. The coagulant mode can be further selected as a pinpoint or spray type in MPC application. In this study the coagulant mode was used for both BPC and MPC dissection; during MPC dissection, the pinpoint application was selected.

For BPC dissection, the power was set at $5 \mathrm{~W}$, and bipolar cautery forceps with a $0.7 \mathrm{~mm}$ fine tip (catalog No. NL 3785-112, V. Mueller/Baxter, McGaw Park, Ill.) were used. The branches were cauterized three times, 1 second per time, by gently squeezing and then reapplying the forceps along a $2 \mathrm{~mm}$ wide zone, which was $2 \mathrm{~mm}$ away from the stem of the ITA external wall. The branch was then cut with scissors in the middle of the cauterized zone (Fig. 1, $A$ ). If there was bleeding, the ends were held with the same forceps for recauterization until the bleeding stopped.

For MPC dissection, the power was set at the minimum level that was effective, $15 \mathrm{~W}$, and the standard cautery pen (blade) was used. As with BPC dissection, the pen was gently and slowly placed on the branch at a point $2 \mathrm{~mm}$ away from the stem of the ITA until the artery was divided (Fig. 1, B). If there was bleeding, the ends were touched 


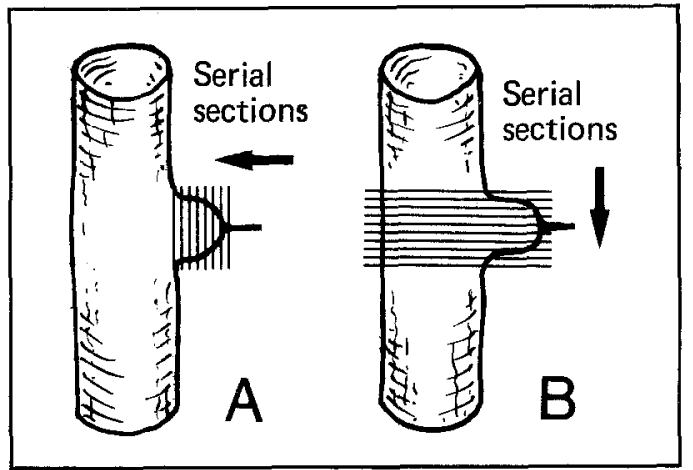

Fig. 2. Orientation of serial sections taken for study. A, Sections taken from cross section of cauterized branch. B, Sections taken from cross section of ITA main stem at branch area.

with the pen for recauterization until the bleeding stopped. After dissection, the ITAs were removed from origin to termination. All dissections were done by one of us (H.Y.) with a uniform surgical technique.

The ITA blood flow was measured in vivo before and after dissection by a Transonic T-208 flowmeter (Transonic System Inc., Ithaca, N.Y.) with a $3 \mathrm{~mm}$ probe. The ITA branches were counted and the ITA length was measured under the following three conditions: in vivo before dissection, in vitro after removal, and in vitro with saline solution inflation at $120 \mathrm{~mm} \mathrm{Hg}$.

The harvested ITAs were then put on a dry sponge and inflated at $120 \mathrm{~mm} \mathrm{Hg}$ with saline solution for 1 minute so that any saline leakage from the branch was absorbed by the sponge and could be weighed for quantitative leakage evaluation. The ITAs were then submerged in a container of saline solution and inflated with air. The inflation pressure was gradually increased to $120 \mathrm{~mm} \mathrm{Hg}$ and the number of branches that showed leakage of air bubbles was noted. This was repeated at $160 \mathrm{~mm} \mathrm{Hg}$ and $200 \mathrm{~mm}$ $\mathrm{Hg}$.

The ITAs were further dynamically inflated and fixed with $10 \%$ formalin at $120 \mathrm{~mm} \mathrm{Hg}$ in a perfusion system that overcame branch leakage and maintained stable pressure for 24 hours; the ITAs were then kept in formalin for an additional week. Two tissue blocks containing the ITA stem and a branch perpendicular to it were taken from each ITA for histologic study. One tissue block was cut into continuous serial cross sections along the branch from the cauterized end toward the ITA stem (Fig. 2, A) to evaluate the extent of the effect of cauterization on the tissue. The other block was prepared with continuous serial cross sections along the ITA stem and across the orifice of the branch to evaluate the integrity of the ITA wall (Fig. 2, $B$ ). In both series, the histologic sections were $6 \mu \mathrm{m}$ thick and were examined at $30 \mu \mathrm{m}$ intervals (approximately 130 sections per specimen). All sections were stained with hematoxylin and eosin (H\&E) stain. In addition, tissue blocks containing the branch that had secondary cauterization because of bleeding from initial cauterization failure were histologically evaluated in a similar manner.
Fifteen ITA pairs were studied with the methods described herein. An additional two pairs were immediately fixed with $3 \%$ glutaraldehyde after removal and flushed with saline solution for scanning electron microscopy (SEM) study (JSM-840A scanning microscope, JEOL Ltd., Tokyo, Japan). Attention was paid to the ITA flow surface, especially at the areas around the internal orifice of the branch. These two ITA pairs did not undergo pressure-leakage tests so that possible surface damage caused by manipulation was avoided.

$\chi^{2}$ or Wilcoxon tests were used for data analysis and statistical comparison.

\section{Results}

The results, including the physical data, the security of branch closure, and pressure-leakage effects from cauterization for both the MPC and BPC groups, are presented in Table I. There were no significant differences between physical aspects of the two groups; however, all the quantitative test results showed the branch closure was significantly more secure in the BPC group.

As shown in Table I, among the 15 ITA study pairs four branches in the BPC group bled after dissection; these branches were all more than $2 \mathrm{~mm}$ in diameter. In contrast, 25 branches in the MPC group, most smaller than $2 \mathrm{~mm}$ in diameter, bled after dissection.

Light microscopy study of H\&E-stained slides showed that there were no remarkable histologic changes in either the wall structure of the ITA stem area or the extreme proximal end of the branch near the ITA stem in either the BPC or MPC groups, except for a few MPC-harvested ITAs that showed intimal corrugation and detachment at the proximal ends of the branches (Fig. 3, $A$ through $D$ ).

There was no significant difference in length between the BPC $(375 \pm 350 \mu \mathrm{m})$ and $\mathrm{MPC}(532 \pm$ $336 \mu \mathrm{m}$ ) groups in the transitional segments in the proximal ends of the branches, for which the wall maintained a relatively normal structural morphology without major destruction from cauterization. Distal to these segments (where the branches were directly cauterized), both the BPC-, and MPCtreated branch walls had similar swelling, intramural hemorrhage, and destruction, with some thrombus in the lumen; however, in many specimens the MPC-treated branches had more wall rupture and more open luminal space than found in the BPCtreated branches (Fig. 3, $E$ and $F$ ). The histologic changes and damage were much more severe and extensive on branches treated with secondary MPC and the damage often extended to the branch orifice 
Table I. Comparison of the effect of BPC and MPC on ITA dissection (15 ITA pairs studied)

\begin{tabular}{|c|c|c|c|}
\hline & $B P C$ & $M P C$ & $p$ Value \\
\hline ITA length before dissection $(\mathrm{cm})$ & $21.4 \pm 1.7$ & $21.1 \pm 1.7$ & $\mathrm{NS}^{*}$ \\
\hline ITA length after removal $(\mathrm{cm})$ & $13.2 \pm 1.5$ & $12.9 \pm 1.0$ & NS* \\
\hline ITA length $(\mathrm{cm})$ inflated at $120 \mathrm{~mm} \mathrm{Hg}$ & $16.5 \pm 1.8$ & $16.3 \pm 1.2$ & NS* \\
\hline ITA blood flow before dissection ( $\mathrm{ml} / \mathrm{min})$ & $45.0 \pm 25.8$ & $44.0 \pm 26.1$ & $\mathrm{NS}^{*}$ \\
\hline ITA blood flow after dissection $(\mathrm{ml} / \mathrm{min})$ & $18.9 \pm 16.4$ & $19.9 \pm 11.9$ & NS* \\
\hline No. of branches & 306 & 279 & \\
\hline No. of leaking branches after initial cauterization & $4(1.3 \%)$ & $25(9.0 \%)$ & $<0.001 \uparrow$ \\
\hline $\begin{array}{l}\text { Amount of saline solution leakage }(\mathrm{ml}) \text { from each ITA } \\
\text { at } 120 \mathrm{~nm} \mathrm{Hg} / \mathrm{min}\end{array}$ & $0.2 \pm 0.3$ & $2.7 \pm 3.2$ & $<0.001^{*}$ \\
\hline \multicolumn{4}{|l|}{ No. of branches with air bubble leakage at } \\
\hline $120 \mathrm{~mm} \mathrm{Hg}$ & $6(2.0 \%)$ & $15(5.4 \%)$ & $<0.05 \dagger$ \\
\hline $160 \mathrm{~mm} \mathrm{Hg}$ & $8(2.6 \%)$ & $28(10.0 \%)$ & $<0.001 \uparrow$ \\
\hline $200 \mathrm{~mm} \mathrm{Hg}$ & $10(3.3 \%)$ & $35(12.5 \%)$ & $<0.001 \dagger$ \\
\hline
\end{tabular}

NS, Not significant.

*Wilcoxon test.

$\dagger \chi^{2}$ test.

area at the ITA stem, whereas secondary BPC caused only slightly more damage than had resulted from primary cauterization and did not affect the ITA stem (Fig. 3, $G$ and $H$ ).

On the flow surface, SEM demonstrated that the lumina of branch orifices treated with BPC were more precise and freer of cellular debris than those of the MPC-treated arteries. Although there was endothelial cell loss around the orificial areas of BPC-treated ITAs, some endothelial cells still remained on the flow surface. However, for the MPCtreated ITAs, there were accumulations of red blood cells and platelets, with almost a complete loss of endothelial cells (Fig. 4, $A$ through $D$ ).

\section{Discussion}

The mechanisms of MPC and BPC electric current conduction are quite different. For MPC, the current flows from the generator to the cautery pen (the active ellectrode) through the subject's body to the inactive dispersive electrode, which is in contact with the subject, and then back to the generator. In $\mathrm{BPC}$, the current flows only from one forceps tip to the other, and a limited amount of tissue is cauterized in between. Because of the difference in the mechanism, the power setting required for BPC is usually much lower than that for MPC. In this study, $5 \mathrm{~W}$ power was used for BPC, compared with $15 \mathrm{~W}$ for MPC. Thus BPC allows control in delicate areas, and the damage is also confined to the tissue between the tips of the cauterization forceps. ${ }^{10,11}$ Because of this, BPC can be used safely much closer to the phrenic nerve than is possible with MPC, which is a consideration of importance in freeing the ITAs high in the chest.
Our SEM study revealed that the ITA flow surface, especially around the branch orifice, had more damage with MPC than with BPC, and there was thrombus formation at the orifice of the branch treated by MPC (Fig. 4). The more flow surface damage with thrombus accumulation, the more the likelihood of luminal occlusion, especially in a lowflow situation. Those changes are probably a result of spreading of the cautery current, and this spreading is more extensive in the MPC mode than in the BPC mode. Lehtola, Verkkala, and Järvinen ${ }^{12}$ have also described similar surface damage caused by cautery current, either through direct contact with the wall or through conduction by metal hemoclips.

Sealing the ends of a divided branch with MPC is mainly achieved by the heat generated from contact of the cautery pen at one side of the branch wall. If the cautery pen cuts through the wall before it is heat-sealed to the other side, bleeding occurs. With $\mathrm{BPC}$, the walls are pressed together and then securely annealed by the current that passes between the opposed tips of the cautery pen (Fig. 1, Fig. 3, $E$ and $F$ ). This explains why, in our study, the branch end closure was far more secure in the BPC group than in the MPC group, even with the use of less power, as shown in Table I. Table I also reflects a greater potential risk of branch leakage in the MPC group after a pressure increase from 120 to $200 \mathrm{~mm}$ $\mathrm{Hg}$ that is significantly higher ( 20 of $264,7.6 \%$ ) than that in the BPC group (4 of $300,1.3 \%$ ). This is of practical importance in regard to the danger of postoperative bleeding from both ends of divided branches.

After initial cauterization, 9\% of the branches in the MPC group bled, compared with $1.3 \%$ in the 

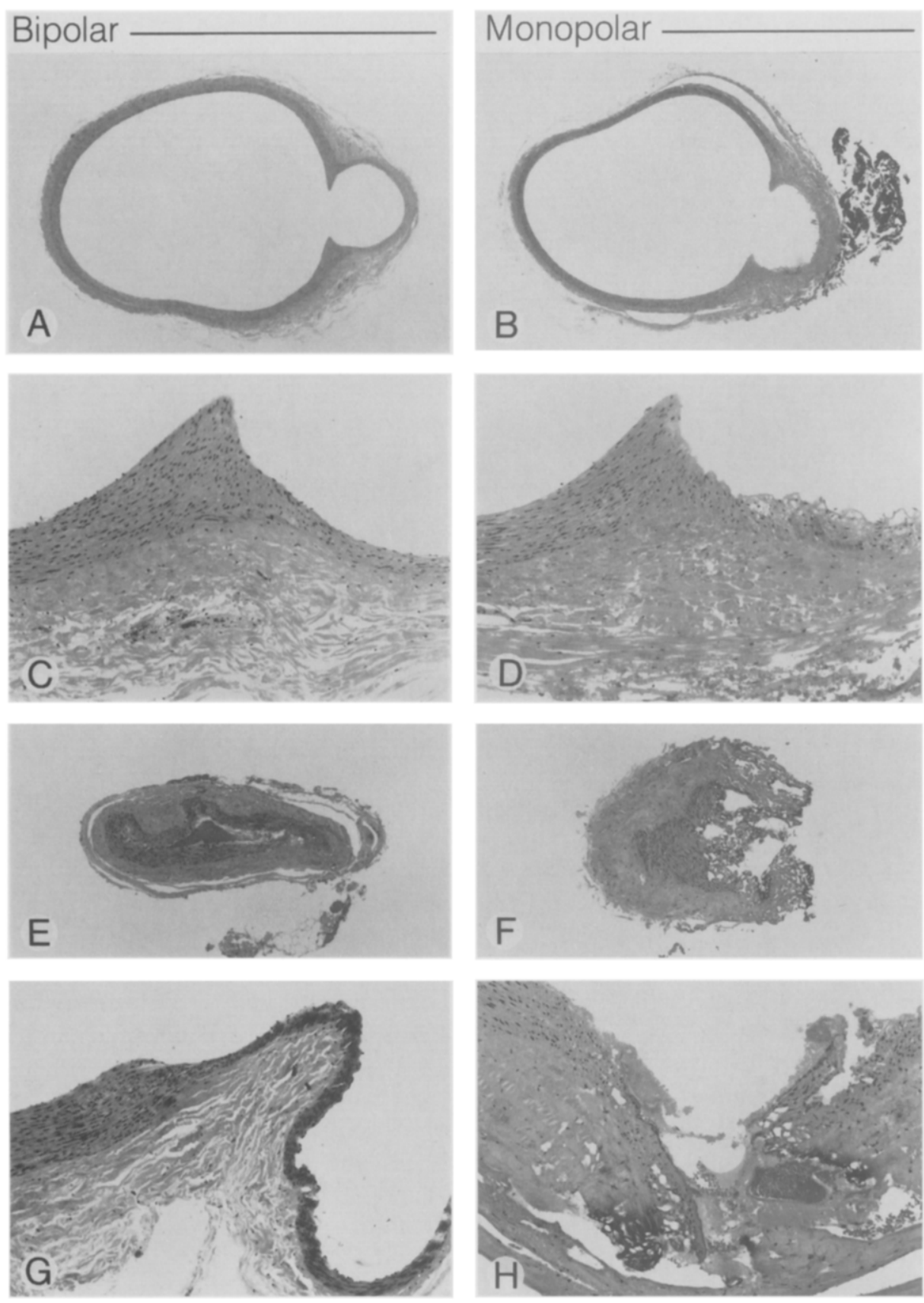

Fig. 3. Light microscopy comparison of histologic study of influence of BPC and MPC on structure of ITA wall. A through D, Cross sections of ITA, including main stem and branch. Luminal area on left, branch area on right. $\mathbf{E}$ and $\mathbf{F}$, Cross section of ITA branch after cauterization. $\mathbf{G}$ and $\mathbf{H}$, Effect of secondary cauterization on wall structure at junction of ITA main stem and branch. Histologically, BPC caused much less change than MPC, both for primary and secondary cauterization ( $\mathrm{H} \& \mathrm{E}$ staining; original magnification of $\mathbf{A}$ and $\mathbf{B} \times 15$, of $\mathbf{C}$ and $\mathbf{D} \times 80$, of $\mathbf{E}$ and $\mathbf{F} \times 35$, of $\mathbf{G}$ and $\mathbf{H} \times 80$ ).

BPC group (Table I). Bleeding from a divided branch end results in subadventitial hematoma. In many instances, this hematoma will cause regional vascular spasm and decrease flow; it may also ob- scure vision needed for a precise secondary cauterization, resulting in unnecessary tissue damage and making an anastomosis more difficult, should it be required in that area. Histologically, severe and 

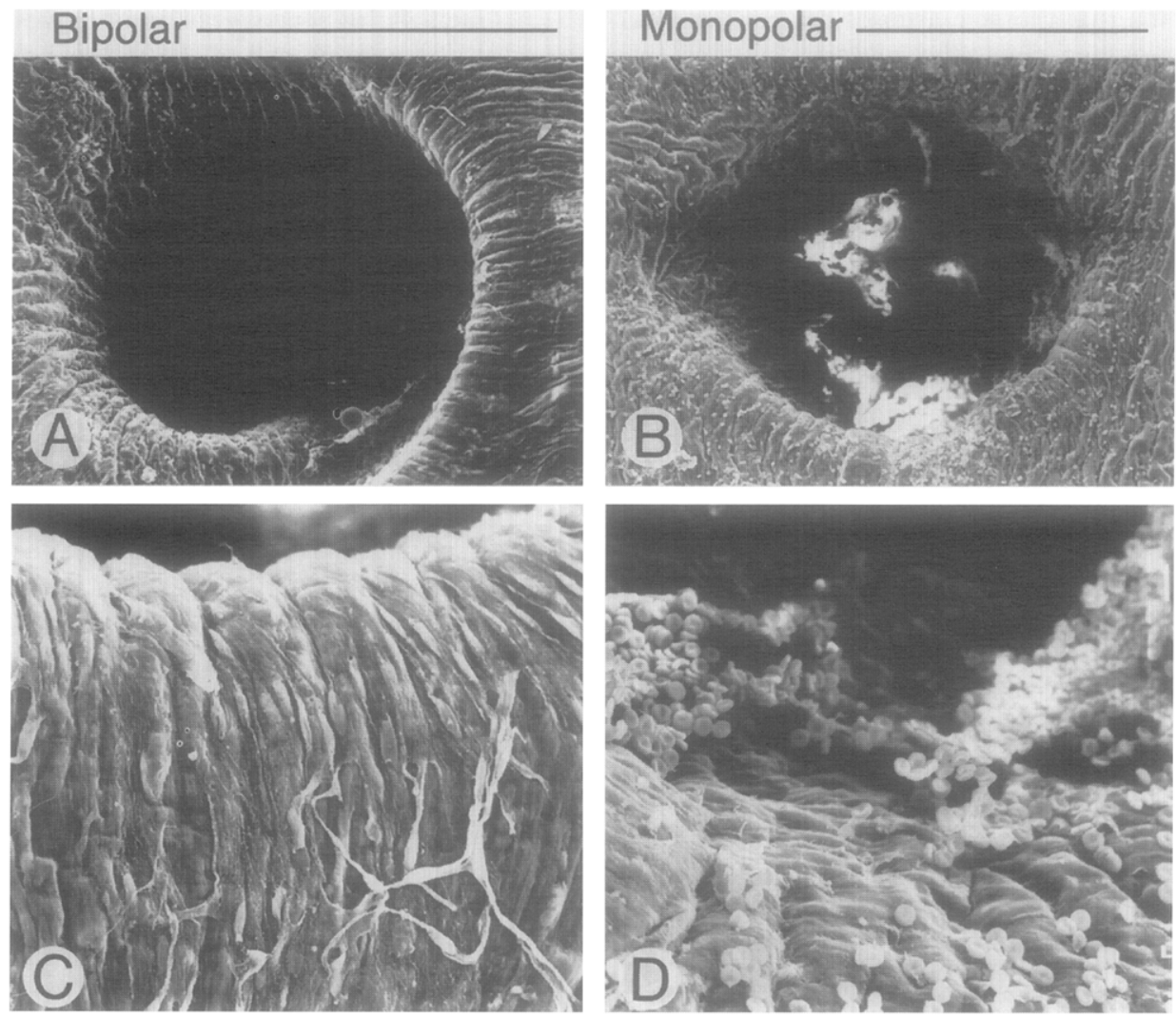

Fig. 4. SEM findings concerning effect of cauterization on flow surface at junction of branch and main stem of ITA. BPC caused less flow surface damage and platelet adhesion than MPC (original magnification of $\mathbf{A}$ and $\mathbf{B} \times 100$, of $\mathbf{C}$ and $\mathbf{D} \times 500$ ).

substantial tissue damage was observed in the ITA wall around the branch orifices after secondary MPC treatment, in contrast to the slight changes observed with BPC (Fig. 3, $G$ and $H$ ). This indicates an additional risk of adverse effects on branches prepared by MPC.

In the BPC group, four branches, all more than $2 \mathrm{~mm}$ in caliber, required secondary cauterization (Table I). This suggests that with BPC, when the branch caliber is greater than $2 \mathrm{~mm}$, each cauterization time should be increased to slightly more than 1 second and the number of applications should also be increased; otherwise hemoclips will be necessary to achieve reliable branch division and closure.

Freeing the ITA as a pedicle must be done with the MPC technique. The BPC technique may be of value in further securing branch closure after pedicle development; however, the use of hemoclips is a reliable means to reinforce branch closure under these circumstances.

The security and safety of mobilizing the ITA in a skeletonized manner is greatly facilitated by use of BPC for secure bonding of the branch walls before they are divided.

This study demonstrates that the use of BPC for skeletonized dissection of the ITA will provide a better flow surface and more secure closure at the divided end of the branch than MPC.

\section{REFERENCES}

1. Loop FD, Lytle BW, Cosgrove DM, et al. Influence of the internal-mammary-artery graft on 10-year survival and other cardiac events. N Engl J Med 1986;314:1-6.

2. Spencer FC. The internal mammary artery: the ideal coronary bypass graft [Editorial]. $\mathrm{N}$ Engl $\mathrm{J}$ Med 1986;314:50-1.

3. Sauvage LR, Wu H-D, Kowalsky TE, et al. Healing basis and surgical techniques for complete revascularization of the left ventricle using only the internal mammary arteries. Ann Thorac Surg 1986;42:449-65.

4. Sauvage IR. Extensive myocardial revascularization 
using only internal thoracic arteries for grafting the anterior descending, circumflex, and right systems. In: Meyers WO, ed. Cardiac surgery: state of the art reviews. Philadelphia: Hanley \& Belfus, 1992:387-419.

5. von Segesser LK. Arterial grafting for myocardial revascularization. Berlin and Heidelberg: SpringerVerlag, 1990:53-62.

6. Landymore RW, Chapman DM. Anatomical studies to support the expanded use of the internal mammary artery graft for myocardial revascularization. Ann Thorac Surg 1987;44:4-6.

7. Huddleston CB, Stoney WS, Alford WC Jr., et al. Internal mammary artery grafts: technical factors influencing patency. Ann Thorac Surg 1986;42:543-9.

8. Noera G, Pensa P, Lodi R, Lamarra M, Biagi B,
Guelfi P. Influence of different harvesting techniques on the arterial wall of the internal mammary artery graft: microscopic analysis. Thorac Cardiovasc Surg 1993;41:16-20.

9. Keeley SB. The skeletonized internal mammary artery. Ann Thorac Surg 1987;44:324-5.

10. Atkinson LJ. Berry and Kohn's operating room technique. 7th ed. St. Louis: Mosby-Year Book, 1992:16972.

11. Edgerton MT. The art of surgical technique. Baltimore: Williams and Wilkins, 1988:95-7.

12. Lehtola A, Verkkala K, Järvinen A. Is electrocautery safe for internal mammary artery (IMA) mobilization: a study using scanning electron microscopy (SEM). Thorac Cardiovasc Surg 1989;37:55-7. 